\title{
Planning of distribution networks considering flexibility of local resources: how to deal with transmission system services Marco Rossi ${ }^{1 *}$, Matteo Rossini ${ }^{1}$, Giacomo Viganò ${ }^{1}$, Gianluigi Migliavacca ${ }^{1}$, Dario Siface ${ }^{1}$, Izabella Faifer ${ }^{1}$, Hakan Hergun ${ }^{2}$, Iver Bakken Sperstad ${ }^{3}$ \\ ${ }^{I}$ Ricerca sul Sistema Energetico - RSE SpA, Milan, Italy \\ ${ }^{2} \mathrm{KU}$ Leuven, Leuven, Belgium \\ ${ }^{3}$ SINTEF Energy Research - SINTEF Energy Research, Trondheim, Norway \\ *marco.rossi@rse-web.it
}

\begin{abstract}
Keywords: DISTRIBUTION NETWORK PLANNING, LOCAL FLEXIBILITY, STORAGE, TRANSMISSION NETWORK PLANNING, ANCILLARY SERVICES
\end{abstract}

\begin{abstract}
Modern planning techniques for distribution systems consider, in addition to the conventional grid reinforcement, the provision of power flexibility from local resources. This solution is demonstrated to be cost-effective in numerous cases. However, distribution resources might be required to provide services to transmission system too, and this aspect needs to be considered within the selection of the best distribution planning options. This paper investigates a distribution network planning strategy based on different trade-offs between "minimization of investment costs" and "maximization of distribution flexibility for transmission services", which is aimed at supporting a cooperative (but decoupled) planning for both distribution and transmission systems.
\end{abstract}

\section{Introduction}

The evolution of power systems introduces new challenges in terms of operation and planning, and current research is demonstrating how local services (provided by flexible demand/generation/storage) can compete with the conventional network reinforcement at any voltage level. In fact, literature [1]-[8] proposes many distribution network planning strategies aimed at determining the best trade-off between local flexibility and new lines/transformers. All of them are clearly showing how the current practices (based on manual procedures and worst-case scenarios analysis) are not leading to optimal solutions. On the contrary, the adoption of dedicated optimization techniques (based on time series processing and multiple scenario analysis [1][2]) assists the selection of the most cost-effective planning option for the solution of local problems.

Nevertheless, distribution network resources have the potential of providing services to the transmission system too and this is a standard requirement already (especially in terms of curtailment of renewable generation [3]). This means that distribution system operators might be required to operate/plan their network in order to guarantee a given amount of local flexibility for transmission services [4]-[6]. However, there is an evident and unexplored conflict between:

- the minimization of the costs related to local congestion management and

- the maximization of the distribution flexibility that can be exploited for transmission services.
For this reason, numerous research initiatives [9][10] and working groups [1][3][5] are suggesting enhanced cooperation among transmission and distribution operators, having the objective of minimizing the operational and planning costs for the entire system [7].

The absolute optimal planning solution, which considers the necessities of the system at any voltage level simultaneously, can be achieved by merging the models of transmission and distribution systems in a single, joint optimization problem. However, in addition to requiring a significant (and probably unbearable) computational burden [2][8], the lack of transparency and standards to exchange information among system operators is one of the barriers for a joint planning of transmission and distribution networks [1].

Having considered the challenges discussed above, this paper proposes a procedure for distribution network planning which, in addition to the solution of local issues, guarantees:

- the consideration of potential transmission system requirements in terms of ancillary services, by trading-off investments cost and exploitable flexibility;

- facilitated negotiation between transmission and distribution operators for the selection of planning options which meet the requirements of both the systems, with no exchange of detailed network information.

\section{Case study and network model}

In order to illustrate the planning strategy, the European configuration for the CIGRE Medium Voltage distribution network benchmark [11] has been adopted as reference. In 
particular, all the switches have been considered to be operated in their normal state and the capacity of the grid lines are selected to be 6.5 MVA for underground cables and 5.5 MVA for overhead lines.

\subsection{Modifications of the standard benchmark case}

The case study focuses on the solution of the planning problem, having considered the availability of local storage units for the provision of congestion management services. For this reason, all the remaining loads and generators are assumed to be non-dispatchable and to feature the 1-day power profiles suggested by [11].

The considered distribution network, in its current configuration, is not subject to any congestion risk. For this reason, the planning problem is studied having supposed an increased wind generation (connected to bus 7), from the existing 1.5 MW to 9 MW. Finally, an additional storage unit is assumed to be connected to bus 14 , such that the entire system features:

- a 0.6 MW - 1.2 MWh storage unit on bus 5;

- a $0.2 \mathrm{MW}-0.4 \mathrm{MWh}$ storage unit on bus 10 ;

- a 1.0 MW - 2.0 MWh storage unit on bus 14 .

All these units are assumed to have a $90 \%$ efficiency during both the charging and discharging phases, and a $0.1 \%$ hourly self-discharge rate.

\subsection{Planning candidates}

The operation with $9 \mathrm{MW}$ wind power is not feasible within the considered system: some lines would be significantly overloaded and voltage problems may occur if not properly managed (reactive power flexibility of generators and tap changing transformers are assumed to be adjustable at no operational cost). In order to face the possible congestions, the following planning candidates have been foreseen:

- Each existing branch can be reinforced by an alternative line/transformer characterized by twice its conductance and capacity (this is equivalent to the installation of two branches in parallel). The cost for line reinforcement is assumed to be equal to:

○ $150 \mathrm{k} € / \mathrm{km}$ for underground cables;

- $60 \mathrm{k€} / \mathrm{km}$ for overhead lines;

- $350 \mathrm{k} €$ for (50 MVA) transformers.

- $\quad$ Each existing storage can be doubled in rated power and capacity, having considered investment costs equal to $350 \mathrm{k} € / \mathrm{MWh}$.

In addition to these candidates, flexibility of existing storage is also considered by the planning procedure. According to that, their related operational costs are included within the objective function and depend on the wholesale energy price, which is assumed to be equal to $50 € / \mathrm{MWh}$ over the entire planning horizon.

The case study is limited to few simple candidates, aimed at returning intuitive and easy to be interpreted results. In fact, the paper focuses on the proof-of-concept for the proposed methodology, which can be anyway applied to more complex situations and sets of possible investments.

\subsection{Model of upgradable distribution network}

The planning of a distribution network consists of the selection of the grid expansion options (new lines and transformers) and flexible units (dispatchable storage) which guarantee the minimum capital and operational expenditures for the solution of existing or potential issues. Therefore, it can be formulated as a classical optimization problem and, since binary investment decision variables needs to be managed, a (mixed-integer) linear formulation is the preferred option [2], especially for large power systems which can be also characterized by a significant amount of expansion candidates.

Given that voltage is one of the electrical quantities to keep under control for distribution network operation and planning [12], a linear approximation of the AC Optimal Power Flow (OPF) is adopted [13]. Thanks to this formulation, which is recognized to be accurate for conventional Medium Voltage distribution grids, a computational efficient algorithm can be easily coded and processed for the planning options described in the following section.

\section{The proposed planning strategy}

As anticipated above, there is conflict between the minimization of the planning costs (limited to distribution network needs) and the delivery of power flexibility to the transmission network. For this reason, the proposed strategy is based on the iterative exploration of a number of possible planning options which cover different trade-offs in terms of costs for local network investments and available capacity for transmission services. Each option consists of the result of more optimization problems, which can be sequenced in different ways. The proposed strategy can be summarized with the three main steps described in the following subsections.

\subsection{Step 1 - Minimization of the planning costs for distribution network}

The most intuitive and common procedure for the planning of distribution system consists of minimizing the operational and investment costs for the solution of local network issues, regardless of transmission potential needs. For the considered case study, the objective function is composed by two terms:

- Storage operation cost, which results from internal losses and energy price. The resulting cost is re-scaled to consider a 10-year lifespan (having assumed the investigated 24-hour time slot to be recurrent).

- Line/transformer/storage investments, which actual costs are reported in section 2.2.

By processing the optimization problem for the considered case study, the returned solution can be summarized as:

- $\quad$ upgrade of the lines connecting buses 3-8 and 7-8;

- operation of existing storage units connected to bus 5 and bus 10, for congestion management services;

- total operational + investment costs of about $245+446 \mathrm{k} €$. 
In fact, looking at the results reported in Figure 1, the loading of the substituted lines is exceeding the initial 6.5 MVA capacity during the wind production peaks.

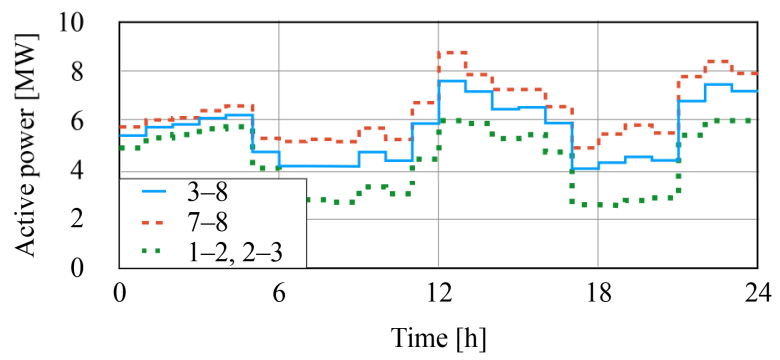

Figure 1. Loading of lines originally subject to congestion risk when step 1 candidates are selected.

Optimization results clearly indicate that also storage units are contributing to the reduction of network issues. Looking at their power exchange profiles (Figure 2), the existing units connected to bus 5 and bus 10 are absorbing active power in order to prevent the congestion of the lines connecting bus 1-bus 2 and bus 2-bus 3 (Figure 1).

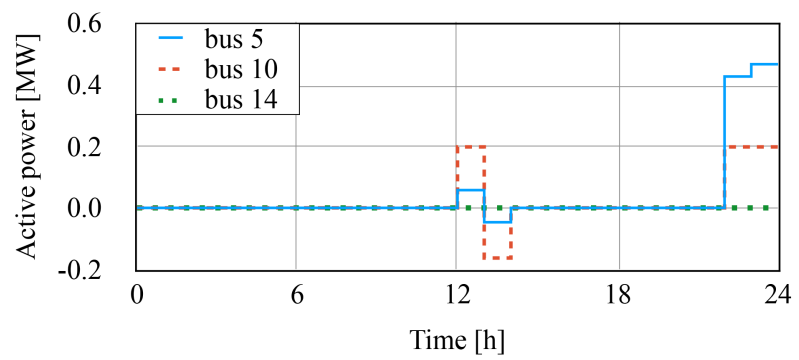

Figure 2. Active power exchanged by existing storage units when step 1 candidates are selected.

Once the optimal investments have been defined, the portion of local flexibility remaining from the local congestion management can be used for services supporting the operation and planning of the upstream transmission network. This portion can be quantified by fixing the selected investments and running two separated OPF routines with the following objective functions:

- maximization of the active power import from transmission to distribution system;

- maximization of the active power export from distribution to transmission system.

Although the implementation of this optimization problem seems to be intuitively correct, the solution of the proposed OPF with these objective functions can be misleading. In fact, the constraints in terms of storable energy limit the maximum power that can be delivered by the storage units in a given time period. Since storage flexibility can be requested anytime for transmission services, the removal of inter-temporal constraints (i.e. energy accumulation) is meaningful for this optimization step. This means that storage units are temporarily modelled as dispatchable devices, featuring the same power capability.

Figure 3 reports the result of these two optimization routines, which makes evident the availability of a power flexibility bandwidth around the baseline profile resulting from the cost-minimization problem.

In order to evaluate, for each storage unit, the portion of available flexibility that can be exploited for transmission services, the power profiles obtained from these three optimization procedures are compared (Figure 4).

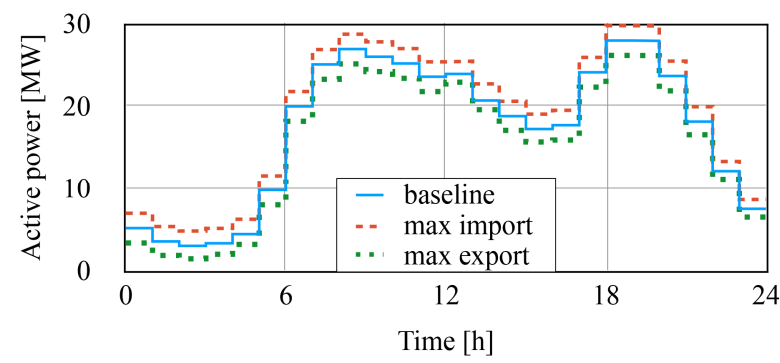

Figure 3. Possible active power exchange profiles between distribution and transmission system when step 1 candidates are selected.

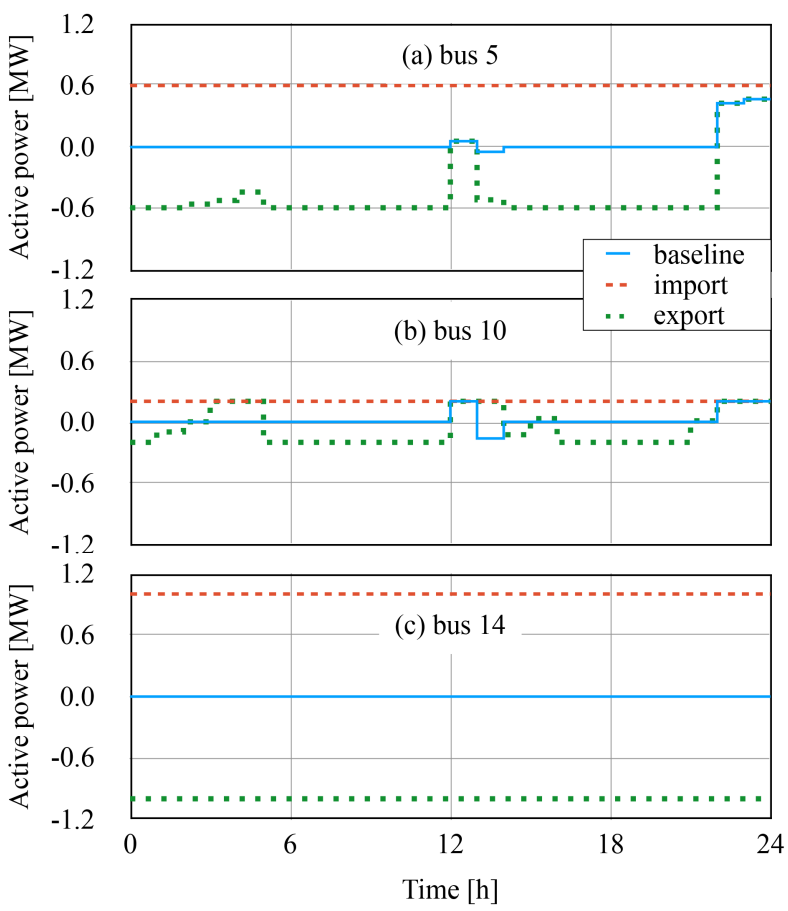

Figure 4. Storage units active power profiles returned by the step 1 of the proposed planning procedure.

From their analysis, it can be clearly recognized that:

- Storage units connected to bus 5 and bus 10 are involved in local services and cannot be considered constantly available for other purposes. In fact, maximum import/export profiles overlap the baseline in case of local congestion.

- Storage unit connected to bus 14, instead, can be exploited in its full power capability, since the obtained profiles result to be flat, i.e. not affected by distribution services and bottlenecks.

In conclusion, having considered that local congestion management is not generally correlated to reserve needs at 
transmission level, the unit connected to bus 14 represents the only reliable resource capable of providing flexibility services to the upstream network. Therefore, in case this planning option is selected, the transmission system would see a single equivalent storage unit, featuring 1.0 MW active power capability and 2.0 MWh storable energy.

\subsection{Step 2 - Maximization of distribution network energy export/import}

Another interesting planning option consists of identifying the network investments capable of maximizing the exploitability of local flexible resources for transmission services. The solution to this problem is obtained by using a similar procedure but reversed with respect to the previous step. In this case, the maximum power export/import profile is defined before the selection of the planning investments, and it is obtained by running (again) two separated OPF routines with the following objective functions:

- maximization of the active power import from transmission to distribution system;

- maximization of the active power export from distribution to transmission system.

Contrarily to step 1, planning candidates are freely selectable within this optimization problem, guaranteeing the maximum power flexibility transfer to the upstream network (at any cost) achieved. Figure 5 demonstrates that a significantly larger regulation bandwidth can be achieved around the baseline profile (which is calculated later in the process).

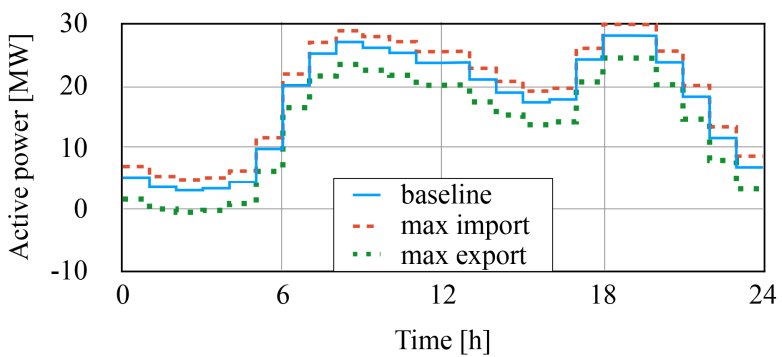

Figure 5. Possible active power exchange profiles between distribution and transmission system when step 2 candidates are selected.

According to the adopted objective functions, investment costs are not influencing the selection of the available candidates. For this reason, some of them can be built even if they are not contributing to the achievement of the maximum power export/import. Therefore, a further OPF problem is solved by minimizing the investment costs, having imposed a power exchange with the transmission network equal to the maximum export/import profile. Thanks to this strategy, unnecessary investments are removed, and for the considered case study the results are:

- $\quad$ upgrade of the lines connecting buses 1-2, 2-3, 3-8, 7-8;

- $\quad$ power/energy upgrade of all existing storage units;

- investment costs equal to $2,792 \mathrm{k} €$.

Until now, step 2 disregards the inter-temporal constraints for the same reasons described in the previous subsection. However, in order to investigate the actual exploitability of storage units for transmission/distribution services, their physical behaviour needs to be fully modelled (including energy capacity limitations, conversion efficiency, etc.). Therefore, having enabled the inter-temporal constraints and fixed the network investments to the ones listed above, a new OPF is carried out with the objective of minimizing the costs related to storage operation. This last run returns the baseline power profile for all the resources when step 2 candidates are selected.

As expected, the increment of investments reduces the necessity of local congestion management, leading to a different absorption/injection of active power for the upgraded storage units (Figure 6).

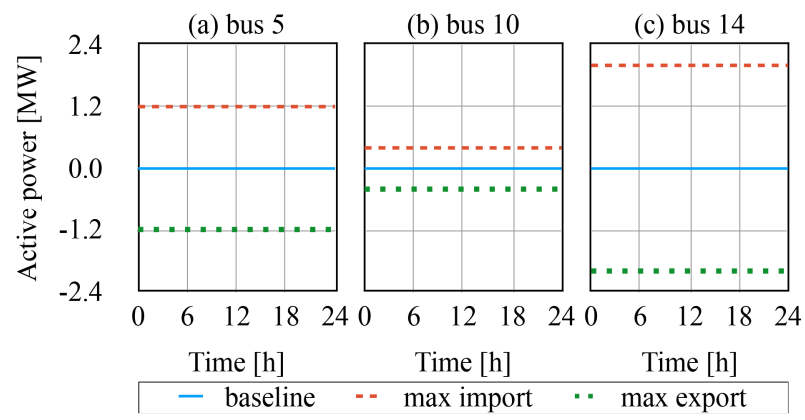

Figure 6. Storage units active power profiles returned by the step 2 of the proposed planning procedure.

The absence of temporary limitations demonstrates the full exploitability of the considered units for transmission network services. This means that, in case this planning option is selected, the transmission system would see a single equivalent storage unit, featuring $3.6 \mathrm{MW}$ active power capability and 7.2 MWh storable energy.

\section{3. $\quad$ Step 3 - Trade-off between minimum costs and maximum flexibility transfer from distribution to transmission network}

Step 1 and step 2 represent two extreme cases in terms of distribution network planning, which lead to significantly different investment decisions and costs. Having considered the number of candidates, it is reasonable to assume that intermediate planning options are existing and their inclusion within the set of choices can increase their granularity (which is beneficial for the optimality of the final planning decision). Reasonable planning option could clearly involve investments which are more expensive than the ones returned by step 1 , while cheaper than the ones of step 2 . According to that, having selected an arbitrary budget for investments within this range of costs, the exact procedure proposed for step 2 is repeated (having added the budget constraint to the considered OPF problems).

For the case study, just one intermediate option (with investments budget set to $1,619 \mathrm{k} €$ ) has been processed and the selected investments consist of:

- $\quad$ upgrade of the line connecting buses 3-8 and 7-8;

- power/energy upgrade of the storage units connected to bus 5 and bus 14 . 
The evaluation of the amount of storage flexibility exploitable for transmission services can be computed as performed in step 2 . In this case, the upgraded capability of the unit connected to bus 14 is fully exploitable. However, it is interesting to notice that the upgrade of the unit connected to bus 5 is not providing apparent benefits. This can be explained by observing that bus 5 is located in a congested area of the distribution network, and the local storage unit can be freely exploited for a limited amount of time. Therefore, this planning option would be seen by the transmission system as an equivalent storage unit, featuring 2.0 MW active power capability and 4.0 MWh storable energy.

Table 1 summarizes the main outcome of the entire distribution planning procedure and highlights the amount of equivalent storage flexibility that can be exploited for transmission services in the considered alternative cases. Of course, the higher the investments budget, the greater is the volume of the exploitable services.

Table 1. Overview of the planning options resulting from the analysis of the case study.

\begin{tabular}{ccc}
\hline Planning option & $\begin{array}{c}\text { Investment } \\
\text { costs }[\mathrm{k} €]\end{array}$ & $\begin{array}{c}\text { Storage flexibility for } \\
\text { transmission services }\end{array}$ \\
\hline step 1 & 446 & $1.0 \mathrm{MW} / 2.0 \mathrm{MWh}$ \\
step 3 & 1,566 & $2.0 \mathrm{MW} / 4.0 \mathrm{MWh}$ \\
step 2 & 2,792 & $3.6 \mathrm{MW} / 7.2 \mathrm{MWh}$ \\
\hline
\end{tabular}

\section{Conclusion}

This work proposes a planning strategy for distribution networks, capable of exploring several planning options in terms of minimization of the operational/investment costs and maximization of local flexibility for the provision of services to the upstream transmission network. Although the procedure is characterized by a non-negligible complexity, its adoption introduces significant advantages for a global optimization of distribution and transmission systems:

- Automatic and independent distribution planning routine, which explores different options in terms of required regulation reserve for transmission services.

- Cooperation between system operators is expected to be simple and efficient, since the identified distribution planning options can be negotiated with a limited exchange of standard and non-sensitive information.

This approach, which is in its early development phases, has the main goal of supporting the decoupling of planning routines for transmission and distribution networks. This increases the computational tractability compared to solving a fully coupled joint optimization problem while still considering the interactions among different voltage levels.

\section{Acknowledgements}

The research leading to this publication received funding from the European Union's Horizon 2020 research and innovation programme under grant agreement no. 863819 .

\section{References}

[1] Silvestro, F., Pilo, F., Araneda, J.C., et al. 'Review of Transmission and Distribution Investment Decision Making Processes under increasing Energy Scenario Uncertainty'. 25th Int. Conf. and Exhibition on Electricity Distribution - CIRED, 2019

[2] Schaefer, F., Menke, J., Marten, F., Braun, M., 'Time Series Based Power System Planning Including Storage Systems and Curtailment Strategies'. 25th Int. Conf. and Exhibition on Electricity Distribution - CIRED, 2019

[3] Joint Working Group CIGRE/CIRED C1.29, 'Planning Criteria for Future Transmission Networks in the Presence of a Greater Variability of Power Exchange with Distribution Systems'. 2017

[4] Pilo, F., Mauri, G., Bak-Jensen, B., Kampf, E., Taylor, J., Silvestro, F., 'Control and automation functions at the TSO and DSO interface - impact on network planning'. 24th Int. Conf. and Exhibition on Electricity Distribution - CIRED, 2017

[5] Pilo, F., Mauri, G., Hanlon, S., et al., 'Control and automation systems for electricity distribution networks of the future - an update on the activities of joint working group CIGRE C6.25/ B5 /CIRED'. 23rd Int. Conf. and Exhibition on Electricity Distribution CIRED, 2015

[6] Kornrumpf, T., Zdrallek, M., Roch, M., et al., 'Flexibility Options for Medium Voltage Grid Planning'. 24th Int. Conf. and Exhibition on Electricity Distribution - CIRED, 2017

[7] EPRI, 'Developing a Framework for Integrated Energy Network Planning (IEN-P)'. California, 2018.

[8] Jendernalik, L., Giavarra, D., Engels, C., et al., 'A Holistic Network Planning Approach: Enhancement of the Grid Expansion using the Flexibility of Network participants'. 24th Int. Conf. and Exhibition on Electricity Distribution - CIRED, 2017

[9] Migliavacca, G., Rossi, M., Siface, D., et al., 'The Innovative FlexPlan Grid-Planning Methodology: How Storage and Flexible Resources Could Help in DeBottlenecking the European System'. Energies 2021, 14(4), 1194

[10] Rossi, M., Migliavacca, G., Viganò, G., et al., 'TSODSO coordination to acquire services from distribution grids: Simulations, cost-benefit analysis and regulatory conclusions from the SmartNet project'. Electric Power Systems Research, Proceedings of the 21st Power Systems Computation Conference (PSCC 2020)

[11]CIGRE Task Force C6.04,'Benchmark Systems for Network Integration of Renewable and Distributed Energy Resources'. 2014

[12] Aigner, C., Witzmann, R., 'Effectivity of Active Voltage Control Concepts in Distribution Grids'. 25th Int. Conf. and Exhibition on Electricity Distribution CIRED, 2019

[13] H2020 EU project FlexPlan, 'Probabilistic optimization of T\&D systems planning with high grid flexibility and its scalability'. 2021 